\title{
Palmprint Authentication System Based on Local and Global Feature Fusion Using DOST
}

\author{
N. B. Mahesh Kumar and K. Premalatha \\ Bannari Amman Institute of Technology, Sathyamangalam, Erode, Tamilnadu 638 401, India \\ Correspondence should be addressed to N. B. Mahesh Kumar; mknbmaheshkumar@gmail.com
}

Received 23 September 2014; Revised 16 December 2014; Accepted 16 December 2014; Published 31 December 2014

Academic Editor: Qiankun Song

Copyright ( 2014 N. B. M. Kumar and K. Premalatha. This is an open access article distributed under the Creative Commons Attribution License, which permits unrestricted use, distribution, and reproduction in any medium, provided the original work is properly cited.

\begin{abstract}
Palmprint is the region between wrist and fingers. In this paper, a palmprint personal identification system is proposed based on the local and global information fusion. The local and global information is critical for the image observation based on the results of the relationship between physical stimuli and perceptions. The local features of the enhanced palmprint are extracted using discrete orthonormal Stockwell transform. The global feature is obtained by reducing the scale of discrete orthonormal Stockwell transform to infinity. The local and global matching distances of the two palmprint images are fused to get the final matching distance of the proposed scheme. The results show that the fusion of local and global features outperforms the existing works on the available three datasets.
\end{abstract}

\section{Introduction}

The unique physical or behavioural characteristics of human beings are of broad interest in the biometrics based methods [1]. In the modern e-world, biometrics has great potentials because of their high accuracy and convenience to use. Researchers and scientists have systematically investigated the use of a number of biometric characteristics like fingerprint $[2-5]$, face $[6]$, iris $[7,8]$, palmprint $[9,10]$, hand geometry [11], hand vein [12], finger knuckle-print [13, 14], voice [15], and ear [16] in the development of computing techniques. The use of applications like automatic personal authentication systems in day-today life results in reliable and effective security control. Recently, hand-based biometrics has been attracting significant attention in the modern eworld.

The region between wrist and fingers is referred to as palmprint. It has features like texture, wrinkles, principle lines, ridges, and minutiae points that can be used for its representation. The critical properties of biometric characteristics such as universality, individuality, stability, and collectability are satisfied by palmprint compared to other biometric traits. It has relatively stable and unique features. It is nonintrusive and data collection is very easy. It needs fewer cooperations to collect data from persons. It provides high efficiency using low resolution images taken from the low cost device. Recently palmprint based recognition systems have received a wide attention from researchers and efforts have been made to build a palmprint based system based on structural features of palmprints such as principle lines, wrinkles, datum points, minutiae points, ridges, and crease points.

Features extracted from the palmprint image can be classified into local and global features. Local features characterize the main topological characteristics of a small part of the trajectory. Global features characterize the relationship of different line segments within a trajectory. The overall content and shape of the objects in the image represent the global features. The specific information in the local regions is defined as local features. The advantages of local features are defined in terms of template size and its discriminability. But the local features also have inevitable drawbacks in practical usage. Due to the sensitivity of the palmprint image, it is difficult to exactly obtain the local features. The performance of the local features degrades due to its poor palmprint quality and capture area. The global features represent the palmprint in a global perspective. Most of the global features are continuous and smooth everywhere expect in some special 


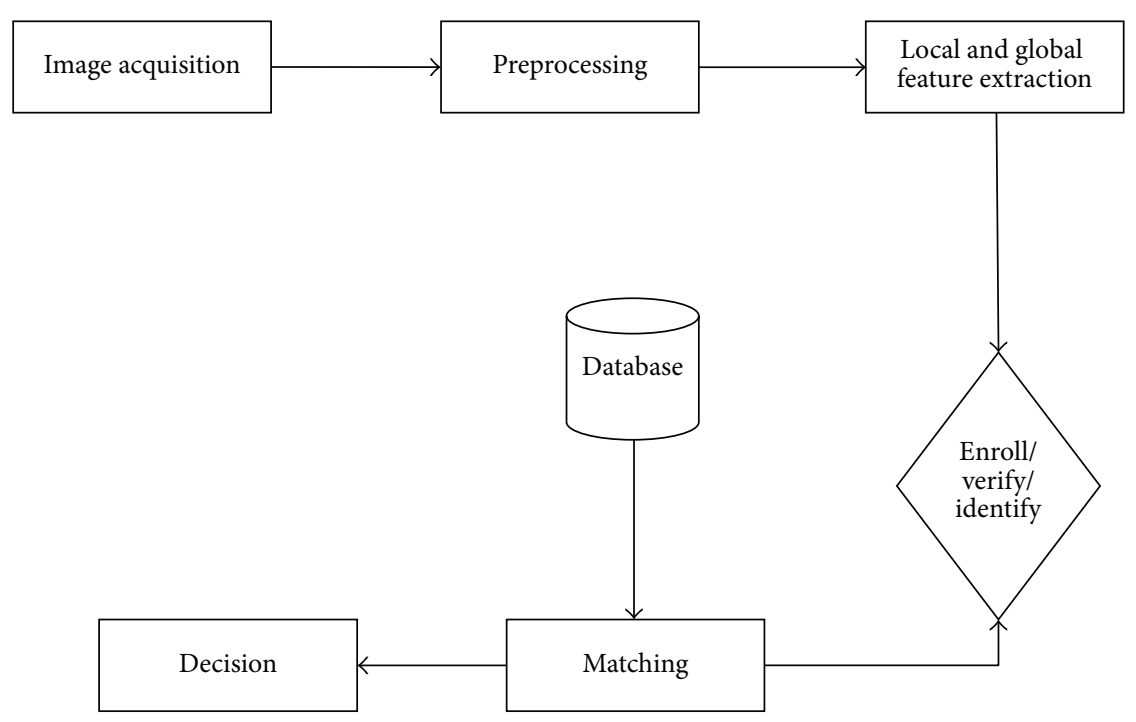

FIGURE 1: Block diagram of the proposed palmprint based recognition system.

regions. The global features can be extracted more consistently from the poor quality of the partial palmprints. It is too space and time consuming to directly store and compare the features pixel by pixel. Global feature based matching overlaps two given templates with different transformation parameters and estimates the similarity score between the corresponding cells. Compared to local features, the global features have less distinctness and hence been often exploited together with other features or in the preprocessing stage of palmprint matching. Both the local and global features are fairly independent and capture current information and hence it is realistic to improve the discriminating ability of matching by fusing features. The local and global features are combined in the matching stage with accessible feature level fusing strategies. The combined local and global features can improve the performance of the palmprint systems on large scale databases. How to select features is pivotal for the effect of feature combination. The combination of irrelative features will improve the accuracy or efficiency of the palmprint biometric systems. The suitable hierarchical approach can be employed to reduce the additional time or memory cost required while fusing the local and global features.

In this work, a local-global feature fusion technique for palmprint biometric system is proposed. The instantaneous phase information is extracted as the local feature by DOST. The global feature can be obtained by aggregating the scale of the DOST. The DOST can be reduced to the Fourier transform of the whole image if the scale of the DOST is increased to infinity. Hence, the local features cannot be obtained but the finest resolution for the global frequency analysis of the image can be obtained. Therefore, Fourier transform coefficients are obviously taken as the global features. The arrangement between intraclass palmprint ROIs can also be refined by the global Fourier features. The two matching distances are calculated by matching the local and the global information separately. Then the two matching distances are merged by fusion rule to get the final matching distance. The block diagram of the proposed system is shown in Figure 1.

The rest of this paper is organized as follows. Section 2 describes the review on palmprint authentication. Section 3 describes the overview of discrete orthonormal Stockwell transform (DOST). Preprocessing and ROI extraction is discussed in Section 4. Section 5 illustrates the local and global feature extraction and matching. Section 6 presents local-global information fusion for palmprint recognition. Experimental results and discussion are demonstrated in Section 7. Section 8 reports the conclusion.

\section{Literature Review}

In [4], a new BioHashing technique is proposed to generate a cancellable template in the context of unordered set of noisy minutiae features and shows that the work is efficient in realistic context. In [17], an intersession variability modelling (ISV) and joint factor analysis (JFA) for face authentication are proposed to achieve a least significant error rate reduction for measuring accuracy. The line features in palmprint are extracted by using sobel and morphological methods [10]. In [18], a new chaff point generation technique for the fuzzy vault in biocryptosystems is proposed and shows that the proposed technique achieves more chaff points compared to Khalil-Hani's algorithm. In [19], the principle lines along with isolated points are used as features. The ridges containing creases of the palmprint are eliminated and it has been proposed in [20]. The features like end points of principle lines have been used in [9]. The end points in the palmprint are found to be directional invariant. The crease points that are related to diseases of a person have been suggested in the palmprint biometrics system. The directional line energy features are also used to identify the palmprint of a person. Further, the palmprint biometric systems that are based on structural features are not invariant to occlusion of the palmprint image. 
The feature extraction and pattern matching of palmprint images are focused mainly. The features in the palmprint images can be classified as local and global features based on the range of pixels involved in the feature extraction. The local feature is a quantity calculated within the local patch and scrambling the detailed behaviours within this specific area. Global information is obtained from all or most of the pixels in the palmprint image and the universal characteristics of the observed image can be examined. Hence, palmprint biometrics systems can be classified into local based approaches and global based approaches according to these definitions. The local-global combination for palmprint recognition has been discussed only in few papers. The local and global features are critical for the image observation and identification of persons as per the theories in the field of psychophysics and neurophysiology [22]. A global feature reproduces the universal features of the image and is appropriate for rough representation. A local feature scrambles the more complete information within the specific region. Therefore, local feature is suitable for better representation. Hence, the improved recognition accuracy is obtained if the local and global feature can be appropriately combined. It is already used in biometrics systems like iris, face, and fingerprint. The conventional Daugman-like classifier uses global features enclosed by zerocrossing boundaries in the palmprint biometrics systems. In two-level palmprint matching scheme [23], the global features for coarse level filtering can be extracted by Hough transform and, for fine-level matching, the local features extracted from the localities and directions of the principal lines in the palmprint.

The local and global features are obtained from the nonnegative matrix factorization technique and principle component analysis in [24] can be combined for palmprint biometric system. In face recognition [6], the global and local information are extracted by principle component analysis (PCA) and Haar wavelets. Global features in the face images are extracted by keeping the low-frequency Fourier coefficients, and the local information is extracted using Gabor filters. The local and global information combination was also utilized in the fingerprint biometrics system [25].

\section{Overview of Discrete Orthonormal Stockwell Transform}

The S-transform is more powerful than other multiresolution techniques like short time Fourier transform (STFT) and wavelet transform (WT). The phase of the S-transform referenced to the time origin provides useful and supplementary information about spectra that is not available from locally referenced phase information in the continuous wavelet transform (CWT).

The S-transform is advantageous for the analysis of palmprint images as it preserves the phase information using linear frequency scaling.

(1) However, the major limitation of S-transform is its high time and space complexity due to its redundant nature, which makes it impractical in many cases.
(2) The 2D-ST of an array of size $N \times N$ has a computational complexity of $O\left(N^{4}+N^{4} \log N\right)$ and storage requirements of $O\left(N^{4}\right)$.

(3) To eliminate this problem of 2D-ST, we use DOST which is also a multiresolution technique for extraction of features from the palmprint images and is based on the S-transform.

(4) DOST have less computational and storage complexity in comparison to the S-transform because it uses an orthonormal set of basis functions, while retaining all the advantageous properties of S-transform.

(5) 2D-DOST provides a spatial frequency representation of an image, with computational and storage complexity as $O\left(N^{2}+N^{2} \log N\right)$ and $O\left(N^{2}\right)$, respectively.

With the dyadic sampling scheme in order $0,1,2 ; \ldots \log _{2} N-$ 1 , DOST of an $N \times N$ palmprint image $f(x, y)$ is performed by the following steps.

(1) Two-dimensional Fourier transform (FT) is applied to the image $f(x, y)$ to obtain Fourier samples $F(u, v)$.

(2) Partition the Fourier sample $F(u, v)$ and multiply it by the square root of the number of points in the partition, and perform an inverse FT. Then the voice image is calculated as

$$
\begin{aligned}
& S\left(x^{\prime}, y^{\prime}, v_{x}, v_{y}\right)=\frac{1}{\sqrt{2^{P_{x}, P_{y}-2}}}, \\
& \sum_{u=-2 p x^{-2}}^{2^{P_{x}-2}-1} \sum_{v=-2^{P_{y-2}}}^{2^{P_{y}-2}-1} F\left(u+v_{x}, v+v_{y}\right), \\
& \text { here } v_{x}=2^{P_{x}-1+2^{P_{x}-2}}, \quad v_{y}=2^{P_{y}-1+2^{P_{y-2}},} \\
& e^{2 \pi i}\left(\frac{u x^{\prime}}{2^{P x-1}}+\frac{v y^{\prime}}{2^{P y-1}}\right),
\end{aligned}
$$

where $v_{x}$ and $v_{y}$ are the horizontal and vertical voice frequencies, where $x^{\prime}$ corresponds to $x^{\prime}$-coordinate in space, where $y^{\prime}$ corresponds to $y^{\prime}$-coordinate in space.

(3) Thus, the features of the palmprint images are obtained after the transformation.

\section{Preprocessing and ROI Extraction}

In this section, the acquired hand image is preprocessed and the palmprint region is extracted. The square area inside the palm region of the hand image is considered as the palmprint or region of interest (ROI). Normally the extracted palm region has the rotation and translation error. In order to minimize the rotation and translation error, the ROI algorithm identifies gaps between fingers. The gaps between the fingers are used as the reference points to align the image. The images of the extracted palm region are aligned rotationally by levelling the gap between the index finger 


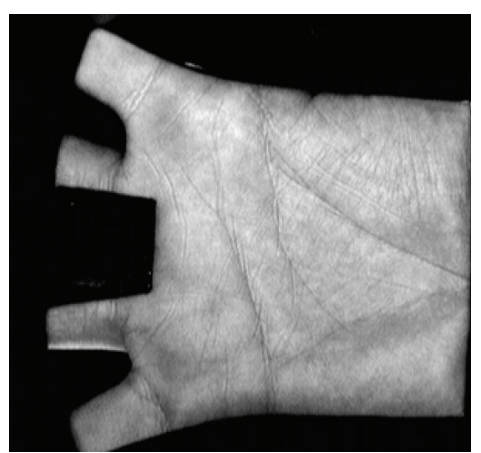

(a)

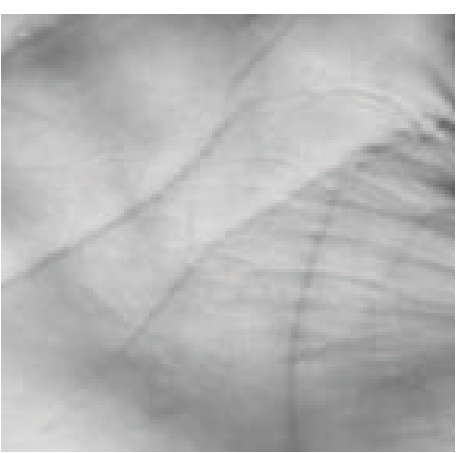

(b)

FIgURE 2: (a) Sample input image. (b) ROI image for PolyU palmprint database.

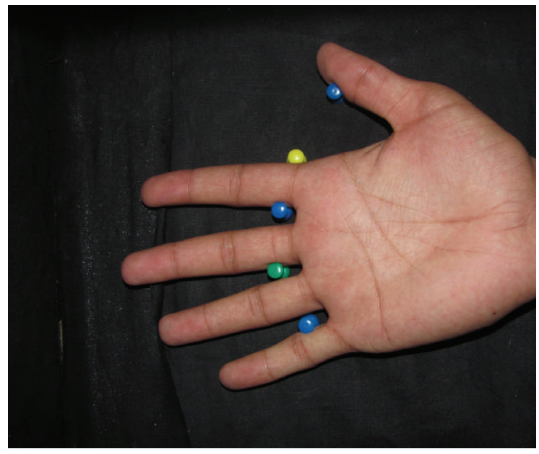

(a)

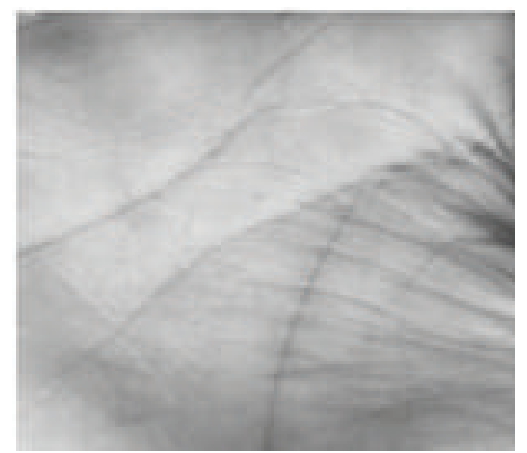

(b)

FIgURE 3: (a) Sample input image. (b) ROI image for COEP palmprint database.

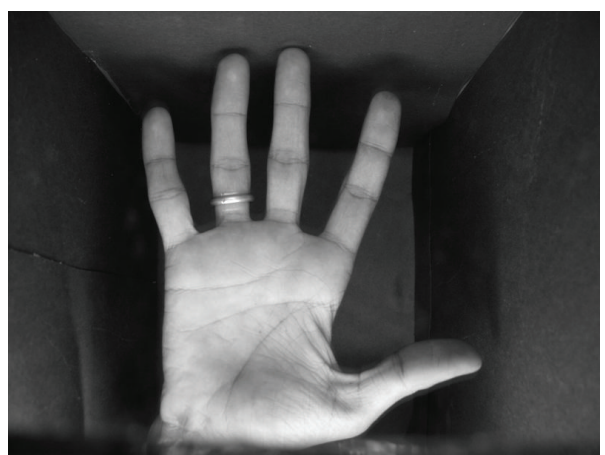

(a)

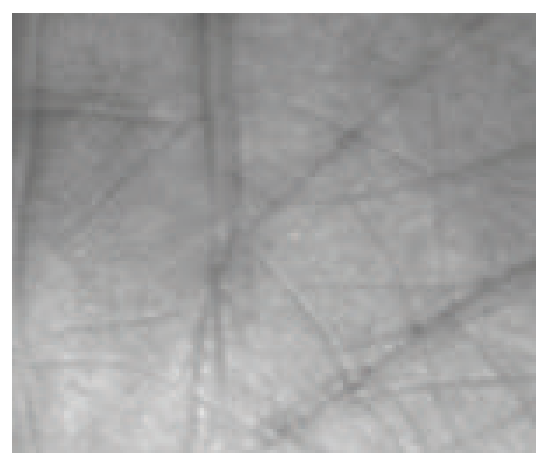

(b)

FIGURE 4: (a) Sample input image. (b) ROI image for IIT Delhi palmprint database.

(IF) and the middle finger (MF) and that between the MF and the ring finger (RF). Among the three gaps, the gap between MF and RF is the toughest point. In order to eliminate the translation error, the gap between MF and RF is used as the reference point. The maximum square region extracted from the palm area is selected as the ROI. The square region is horizontally centered on the axis running through the gap between MF and RF. Due to the regular and controlled uniform illumination conditions during image capture, the acquired hand image and its background contrast in colour. The sample input image is shown in Figures 2(a), 3(a), and 4(a) for PolyU palmprint database, COEP palmprint database, and IIT Delhi palmprint database. Global thresholding has been applied to extract the hand from the background. The image is processed with opening and closing morphological operations to remove any isolated small blobs or holes. Contour-tracing algorithm is applied to extract the contour of the hand image from the palmprint. The ROI extraction technique of IIT Delhi palmprint database, PolyU palmprint database, and COEP palmprint database are same as in $[9,26]$. The corresponding Figures 2(b), 3(b), and 4(b) are shown in this paper which gives the details of ROI image. 


\section{Local-Global Feature Extraction and Matching}

5.1. Local Feature. The feature extraction technique in any palmprint biometric system is used to obtain good interclass separation in minimum time. The features of palmprint are extracted by using 2D-DOST after the completion of preprocessing and ROI segmentation from palmprint. The local variation of instantaneous phase is used to extract features from palmprint. Instantaneous phase obtained using DOST is the resolution of phase with respective to time. It has the merits of great accurateness, sturdiness to brightness variation, and fast matching. When matching two palmprint images, the angular distance based on the normalized Hamming distance is used.

5.2. Global Feature. DOST is used to extract the instantaneous phase information and also it can be considered as a windowed Fourier transform. The DOST can be considered as a windowed Fourier transform. The phase-only correlation (POC) and band-limited phase-only correlation (BLPOC) are utilized to match the global information of the two palmprint images.

5.2.1. Phase-Only Correlation (POC). The Fourier transform coefficients are considered as the global information. The two given Fourier transforms are matched using POC. POC based techniques have been widely used in image registration tasks. Recently, a POC technique considered as a resemblance measure in certain biometrics systems [7, 22]. BLPOC is more effective when compared to the conventional POC. BLPOC is utilized to estimate the translation parameters between palmprint ROIs and also measure the resemblance of the Fourier transforms of the aligned ROIs. POC is an operational method to estimate the displacement parameters between two palmprint images in the Fourier transform domain. Its primary principle is the translation property of the Fourier transforms.

Consider two $N_{1} \times N_{2}$ images $f\left(n_{1}, n_{2}\right)$ and $g\left(n_{1}, n_{2}\right)$, where the assumption is that the index ranges are $n_{1}=$ $-M_{1}, \ldots, M_{1}\left(M_{1}>0\right)$ and $n_{2}=-M_{2}, \ldots, M_{2}\left(M_{2}>0\right)$. For mathematical simplicity $N_{1}=2 M_{1}+1$ and $N_{2}=$ $2 M_{2}+1$. Let $F\left(k_{1}, k_{2}\right)$ and $G\left(k_{1}, k_{2}\right)$ denote the 2D discrete Fourier transforms (2D DFTs) of the two images. $F\left(k_{1}, k_{2}\right)$ and $G\left(k_{1}, k_{2}\right)$ are given by

$$
\begin{aligned}
& F\left(k_{1}, k_{2}\right)=\sum_{n_{1}, n_{2}} f\left(n_{1}, n_{2}\right) W_{N_{1}}^{k_{1} n_{1}} W_{N_{2}}^{k_{2} n_{2}}=A_{F\left(k_{1}, k_{2}\right) e^{j \theta_{F}\left(k_{1}, k_{2}\right)},} \\
& G\left(k_{1}, k_{2}\right)=\sum_{n_{1}, n_{2}} g\left(n_{1}, n_{2}\right) W_{N_{1}}^{k_{1} n_{1}} W_{N_{2}}^{k_{2} n_{2}}=A_{G\left(k_{1}, k_{2}\right) e^{j \theta_{G}\left(k_{1}, k_{2}\right)},}
\end{aligned}
$$

where $k_{1}=-M_{1}, \ldots, M_{1} \cdot k_{2}=-M_{2}, \ldots, M_{2}, W_{N_{1}}=$ $e^{-j\left(2 \pi / N_{1}\right)} \cdot W_{N_{2}}=e^{-j\left(2 \pi / N_{2}\right)}$ and the operator $\sum_{n_{1} n_{2}}$ denotes $\sum_{n_{1}=-M_{1}}^{M_{1}} \sum_{n_{2}=-M_{2}}^{M_{2}} \cdot A_{F\left(k_{1}, k_{2}\right)}$ and $A_{G\left(k_{1}, k_{2}\right)}$ are amplitude components and $e^{j \theta_{F}}\left(k_{1}, k_{2}\right)$ and $e^{j \theta_{G}}\left(k_{1}, k_{2}\right)$ are phase components.

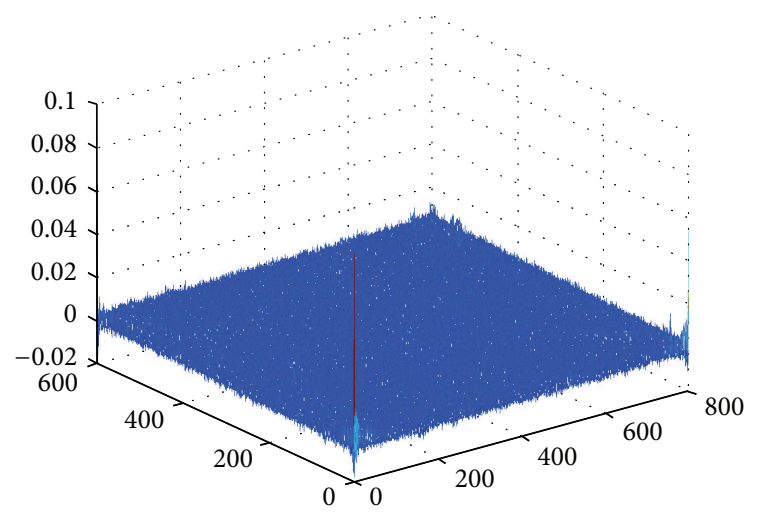

FIgURe 5: POC function for PolyU palmprint database.

The cross spectrum $R\left(k_{1}, k_{2}\right)$ between $F\left(k_{1}, k_{2}\right)$ and $G\left(k_{1}, k_{2}\right)$ is given by

$$
\begin{aligned}
R\left(k_{1}, k_{2}\right) & =F\left(k_{1}, k_{2}\right) \overline{G\left(k_{1}, k_{2}\right)} \\
& =A_{F\left(k_{1}, k_{2}\right)} A_{G\left(k_{1}, k_{2}\right)} e^{j \theta\left(k_{1}, k_{2}\right)},
\end{aligned}
$$

where $\overline{G\left(k_{1}, k_{2}\right)}$ denotes the complex conjugate of $G\left(k_{1}, k_{2}\right)$ and $\theta\left(k_{1}, k_{2}\right)=\theta_{F}\left(k_{1}, k_{2}\right)-\theta_{G}\left(k_{1}, k_{2}\right)$. On the other hand, the cross spectrum (or normalized cross spectrum) $\widehat{R}\left(k_{1}, k_{2}\right)$ is defined as

$$
\begin{aligned}
\widehat{R}\left(k_{1}, k_{2}\right) & =\frac{F\left(k_{1}, k_{2}\right) \overline{G\left(k_{1}, k_{2}\right)}}{\left|F\left(k_{1}, k_{2}\right) \overline{G\left(k_{1}, k_{2}\right)}\right|} \\
& =e^{j \theta\left(k_{1}, k_{2}\right)} .
\end{aligned}
$$

The POC function $\widehat{r_{f}}\left(n_{1}, n_{2}\right)=$ is $2 \mathrm{D}$ inverse discrete Fourier transforms (IDFT) of $\widehat{R}\left(k_{1}, k_{2}\right)$ and is given by

$$
\widehat{r}\left(n_{1}, n_{2}\right)=\frac{1}{N_{1}, N_{2}} \widehat{R}\left(k_{1}, k_{2}\right) W_{N_{1}}^{-k_{1} n_{1}} W_{N_{2}}^{-k_{2} n_{2}},
$$

where $\sum_{k_{1} k_{2}}$ denotes $\sum_{k_{1}=-M_{1}}^{M_{1}} \sum_{k_{2}=-M_{2}}^{M_{2}}$.

The most significant property of POC is compared with the normal correlation and it gives accuracy in pattern matching. The distinct sharp peak is obtained when POC function is the same. The peak in the POC drops considerably if the two palm print images are not the same. Figures 5, 6, and 7 show the POC function of ROI for PolyU palmprint database, COEP palmprint database, and IIT Delhi palmprint database. Thus, the POC function shows much higher perception ability than the normal correlation function. In order to match the two palmprint images, the height of the peak will be used as a good resemblance measure.

5.2.2. Band-Limited Phase-Only Correlation (BLPOC). In the POC based image matching method, all the frequency components are involved. However, high frequency components can be susceptible to noise. BLPOC is used to eliminate noisy high frequency components. BLPOC bounds 


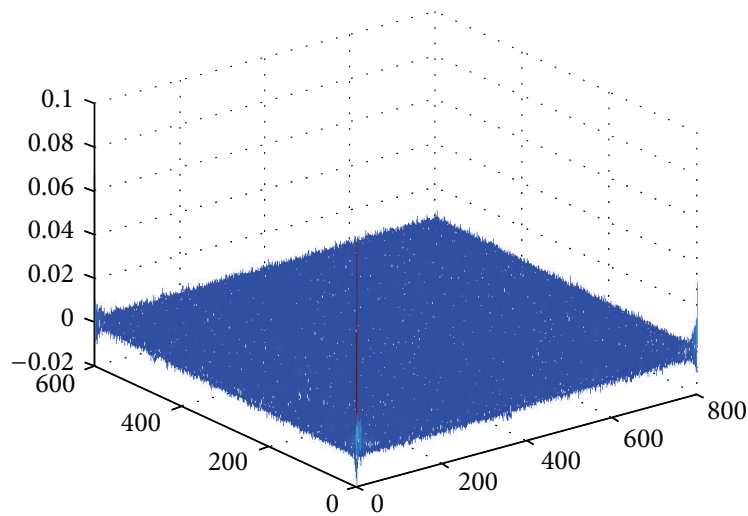

FIgURE 6: POC function for COEP palmprint database.

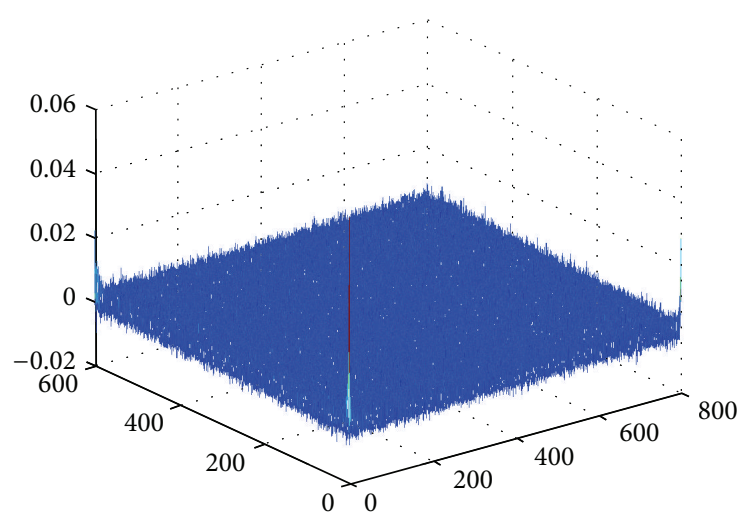

FIgURE 7: POC function for IIT Delhi palmprint database.

the range of spectrum of the given palmprint image. BLPOC function between two ROI images can be measured as the POC function between their low-pass filtered versions based on the definition of BLPOC. Therefore, BLPOC function can preserve the properties of the POC function. BLPOC function got a distinct peak if two ROI images are the same. But the locality of peak in two ROI images gives the translational displacement. Experimentations specify that BLPOC function provides a much higher perception ability than the original POC function in palmprint biometric system. Therefore, the efficient size of spectrum is given by $L_{1}=2 U_{0}+1$ and $L_{2}=2 V_{0}+1$. BLPOC function is given by

$$
p_{g f}^{U_{0} V_{0}}(m, n)=\frac{1}{L_{1} L_{2}} \sum_{u=-U_{0}}^{U_{0}} \sum_{v=-V_{0}}^{V_{0}} R_{G F(u, v)} e^{j 2 \pi\left(m u / L_{1}+n v / L_{2}\right)}
$$

where $m=-U_{0}, \ldots, U_{0}$, and $n=-V_{0}, \ldots, V_{0}$, where $u=$ $-U_{0}, \ldots, U_{0}$, and $v=-V_{0}, \ldots, V_{0}$, where $0 \leq U_{0} \leq M_{0}$, $0 \leq V_{0} \leq N_{0}$.

Figures 8, 9, and 10 are BLPOC function for PolyU palmprint database, COEP palmprint database, and IIT Delhi palmprint database, respectively. These examples indicate that in the instance of genuine matching (a matching performed between a pair of palmprint images from the same hand) the BLPOC will show a much sharper peak than POC;

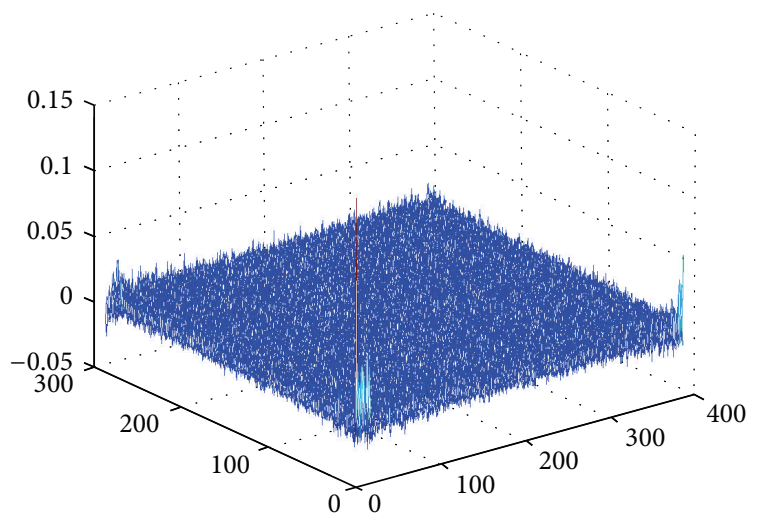

Figure 8: BLPOC function for PolyU palmprint database.

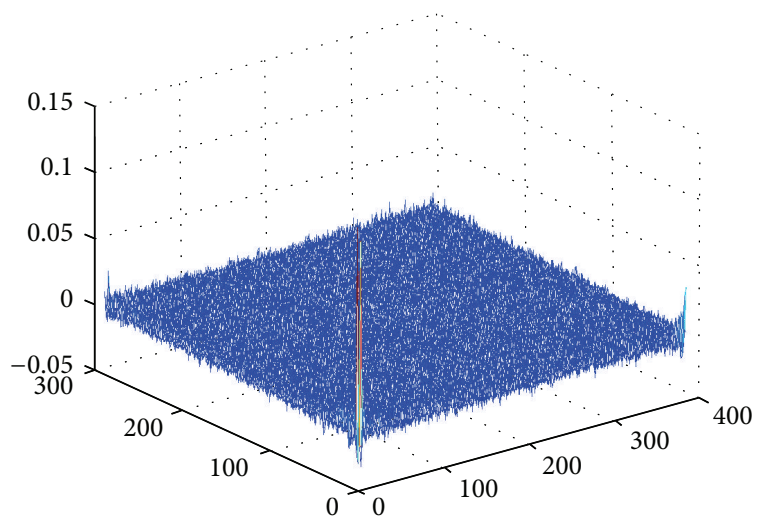

FIGURE 9: BLPOC function for COEP palmprint database.

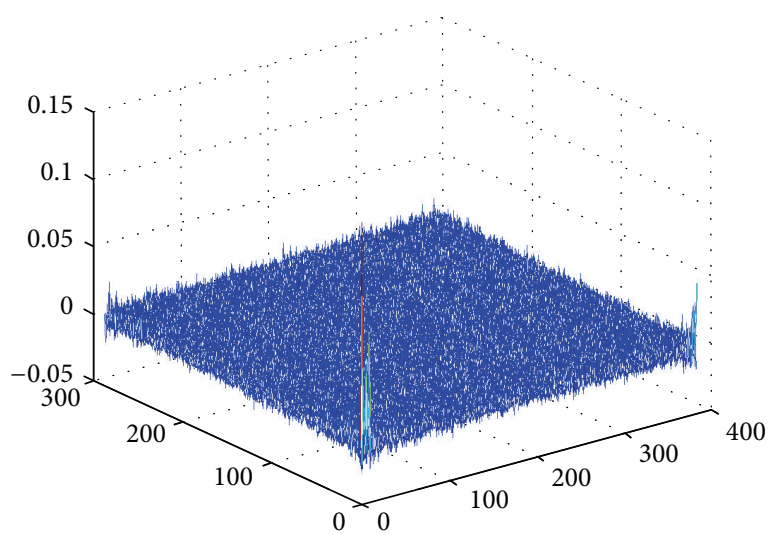

Figure 10: BLPOC function for IIT Delhi palmprint database.

however, for an imposter matching (a matching performed between a couple of palmprint images from different hands), neither BLPOC nor POC will display a different severe peak. BLPOC function can be adopted to align the translation between two palmprint ROI images and then to measure the resemblance between Fourier transforms of the aligned ROIs. 


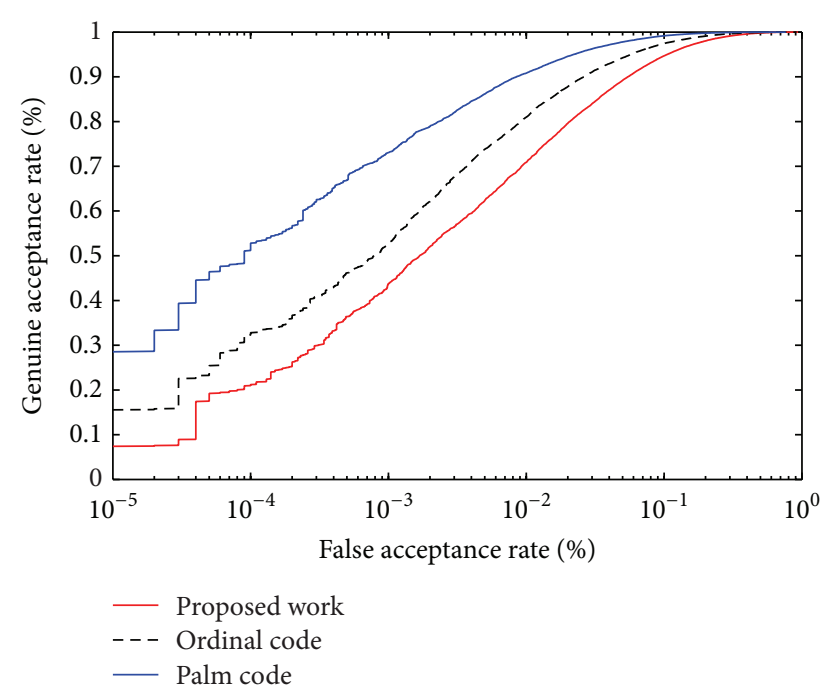

FigURE 11: ROC curve using local features of proposed DOST for PolyU palmprint database.

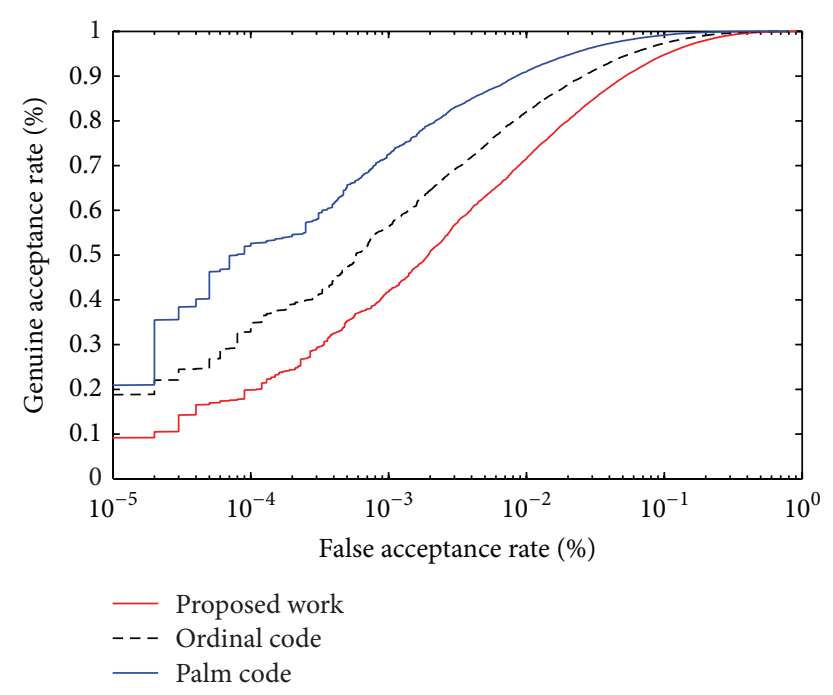

FIGURE 12: ROC curve using global features of proposed DOST for PolyU palmprint database.

\section{Local-Global Information Fusion (LGIF) for Palmprint Recognition}

LGIF based palmprint recognition algorithm is described in this section. The palmprint image acquisition device and the ROI extraction system can decrease the symmetrical transformations between intraclass ROIs. But it is quiet unavoidable because there is some translation between intraclass ROIs in the palmprint images. These will deteriorate the genuine matching scores. This problem is solved by transforming the given information in horizontal and vertical directions numerous times and the smallest of the subsequent similar distances is measured as the final matching distance. This problem can be solved in a dissimilar way by calculating the displacement parameters between two ROIs using BLPOC

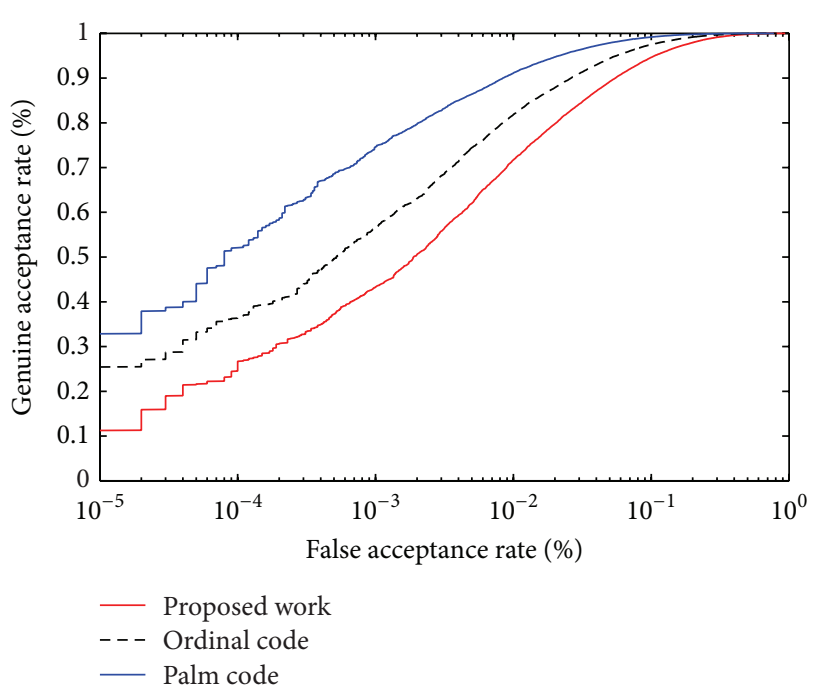

FIGURE 13: ROC curve using local features of proposed DOST for COEP palmprint database.

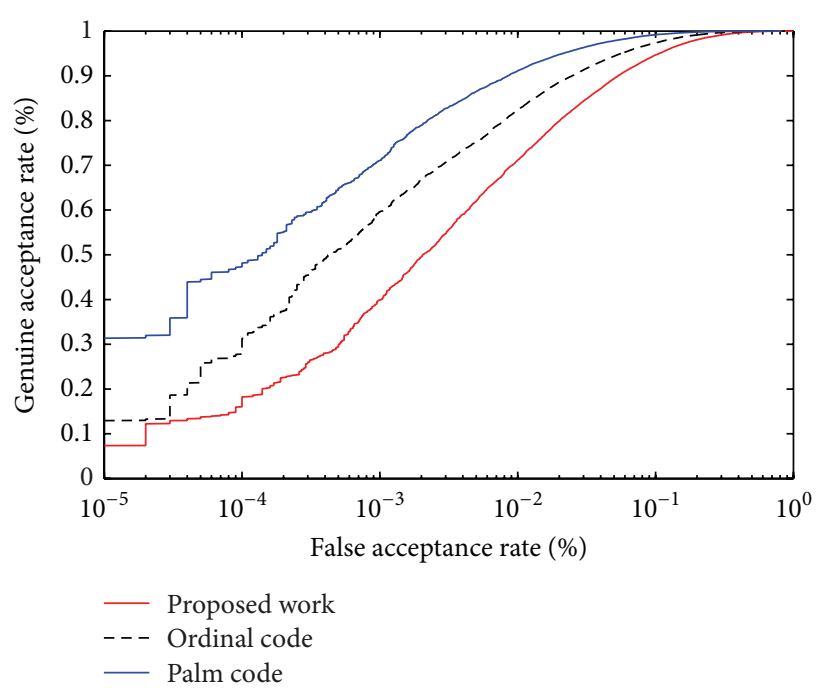

FIGURE 14: ROC curve using global features of proposed DOST for COEP palmprint database.

function. Then the mutual regions can be cropped based on which the pattern matching is completed. The displacement parameters between two palmprint ROI images can be calculated from the peak locality of global BLPOC function. Then two palmprint ROI images can be aligned based on the displacement parameters and extract the mutual regions. Ratio between the mutual region area and the area of the original ROI can be checked in the palmprint system. After alignment and common area cropping, two palmprint ROI images are constructed. Then matching distance is obtained by matching two palmprint ROI images. The angular distance based on the normalized Hamming distance is used to match the two palmprint images for local features in palmprint biometric systems. The two palmprint images $C_{f}$ and $C_{g}$ are created after alignment and common area cropping. The matching 


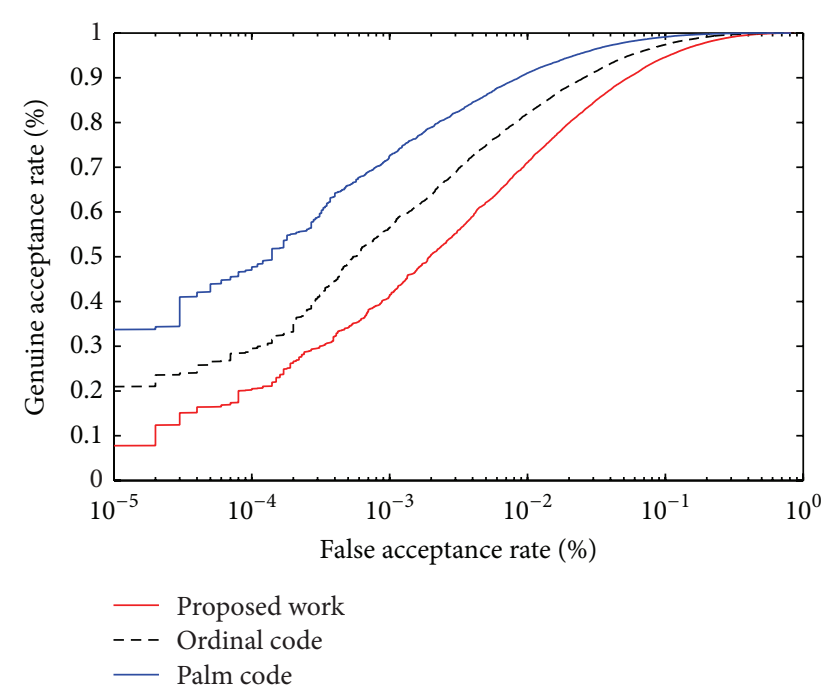

FIGURE 15: ROC curve using local features of proposed DOST for IIT Delhi palmprint database.

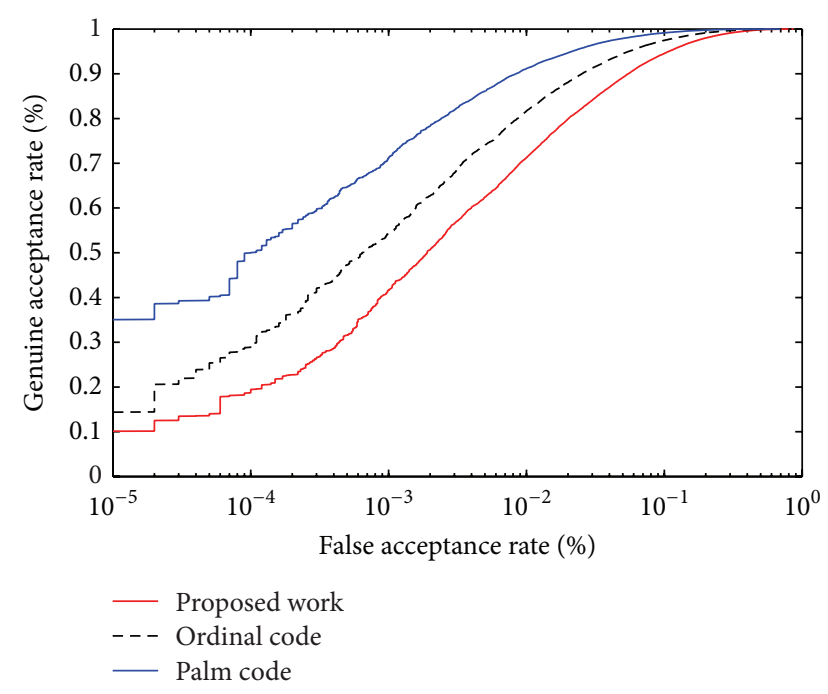

FIGURE 16: ROC curve using global features of proposed DOST for IIT Delhi palmprint database.

distance $d_{L}$ is obtained by matching the two palmprint images $C_{f}$ and $C_{g}$. The peak value of the BLPOC function between two palmprint images is used to measure the similarity of their Fourier transforms. The peak value is indicated as pocS; therefore, the matching distance is termed as $d_{G}=1-$ pocS. The two matching distances $d_{L}$ and $d_{G}$ are obtained. We got the final matching distance by fusing the two distances $d_{L}$ and $d_{G}$. In BLPOC function, the peak value between palmprint ROI images is used to calculate the resemblance of their Fourier transforms to obtain matching distance. In this paper, two matching distances are obtained from two dissimilar matchers; namely, local feature based matcher (matcher 1) and global feature based matcher (matcher 2) and the maximum weighted (MW) rule can be adopted. The weights are assigned according to the equal error rate

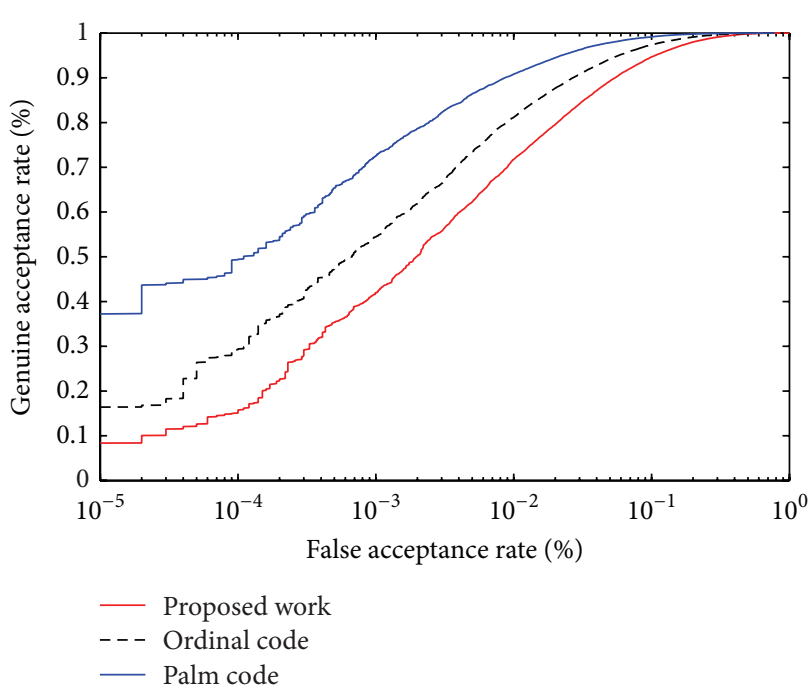

FIgURE 17: ROC curve using both local and global features of proposed DOST for PolyU palmprint database.

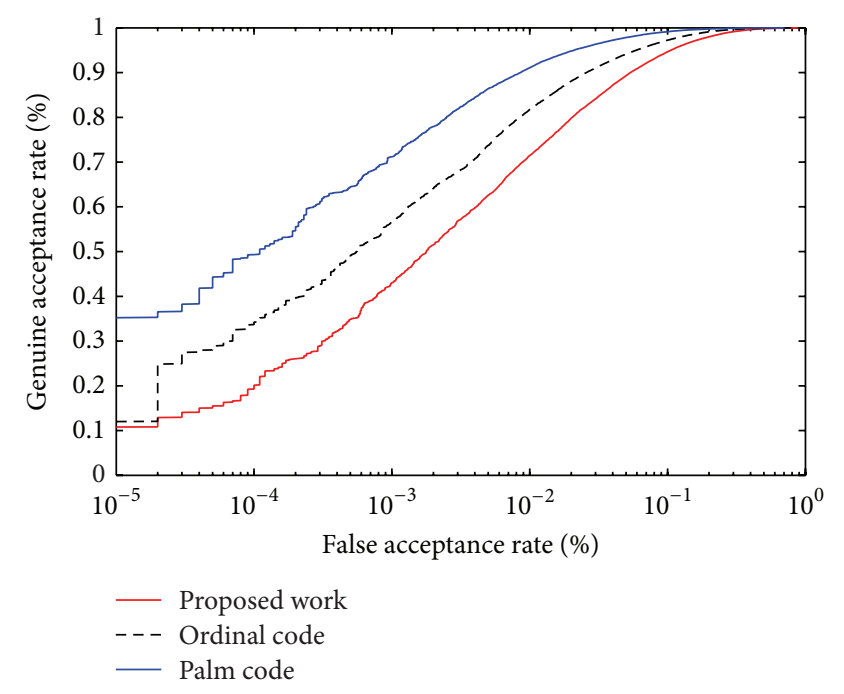

FIgURE 18: ROC curve using both local and global features of proposed DOST for COEP palmprint database.

(EER) obtained on a training dataset by different matchers based on MW fusion rule. It is obvious that the weights are inversely proportional to the corresponding EERs. Then, the final matching distance is estimated.

\section{Experimental Results and Discussion}

Three different sets of hand image databases are used for the proposed system. The PolyU palmprint database [27] contains 7752 grayscale images corresponding to 386 different palms in BMP image format. The twenty samples are collected in two different sessions. 10 samples were collected from first session and 10 samples were captured in the second session, respectively. 


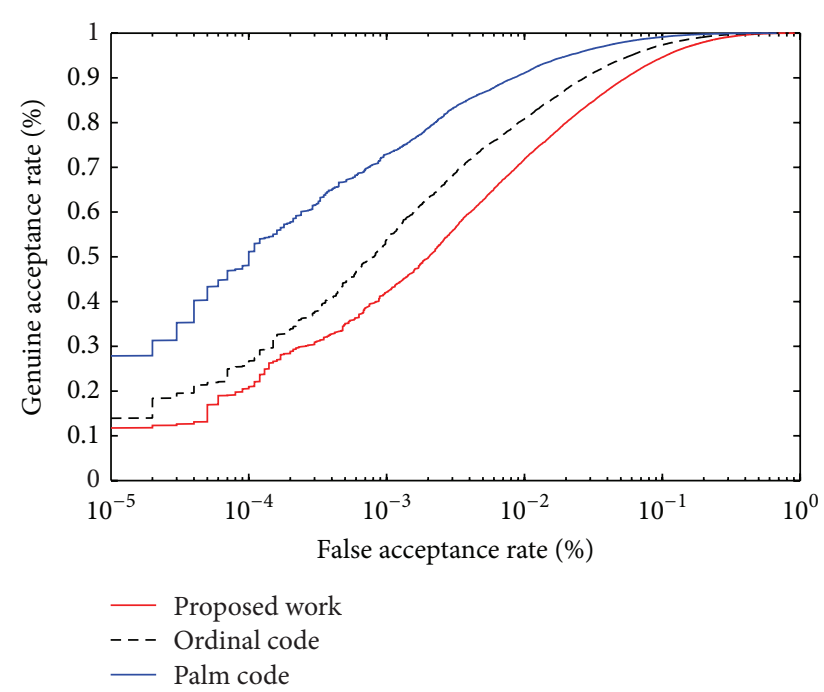

Figure 19: ROC curve using both local and global features of proposed DOST for IIT Delhi palmprint database.

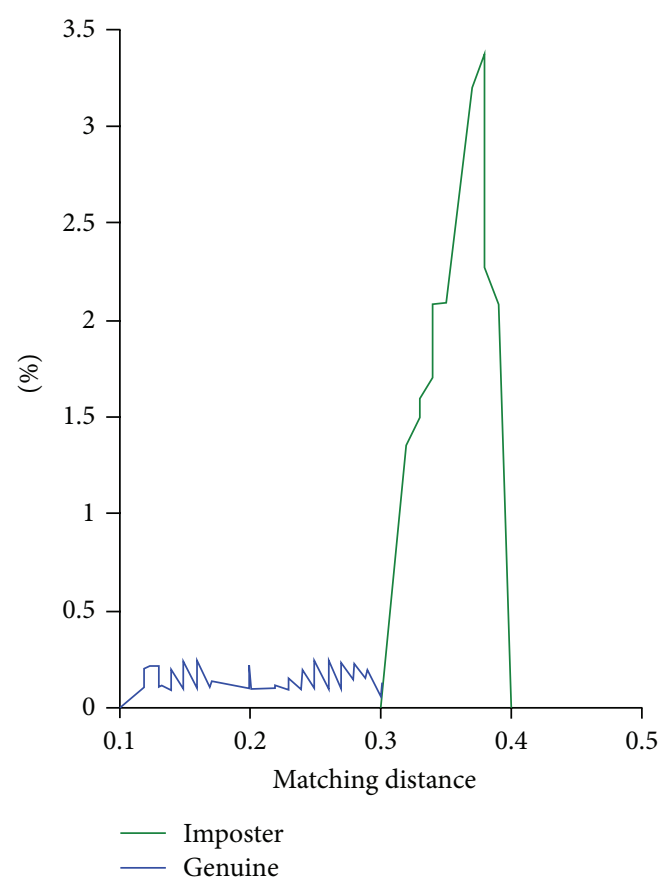

FIGURE 20: Distance distributions of genuine matching and imposter matching of proposed DOST for PolyU palmprint database.

COEP palmprint database contains [28] 1344 images corresponding to 168 people. Eight different images are collected from single person's palm.

IIT Delhi palmprint database [29] contains 230 subjects. The subjects in the palmprint database are collected from the age group 12-57 years. Seven images per each subject are collected from the left and right hand. An identification
TABLE 1: Performance comparison of different palmprint verification schemes for both local and global combination.

\begin{tabular}{lccc}
\hline & CRR (\%) & EER (\%) & DI \\
\hline \multicolumn{4}{c}{ (a) Performance for PolyU database } \\
Palm code [9] & 99.91 & 0.5338 & 5.5807 \\
Ordinal code [21] & 100.00 & 0.0709 & 6.6785 \\
Proposed & $\mathbf{1 0 0 . 0 0}$ & $\mathbf{0 . 0 0 3 5}$ & $\mathbf{7 . 2 5 4 1}$ \\
\hline \multicolumn{4}{c}{ (b) Performance for COEP database } \\
Palm code [9] & 99.61 & 3.6732 & 3.0734 \\
Ordinal code [21] & 99.84 & 1.7544 & 3.3966 \\
Proposed & $\mathbf{1 0 0 . 0 0}$ & $\mathbf{1 . 2 6 0 5}$ & $\mathbf{4 . 0 2 2 8}$ \\
\hline \multicolumn{4}{c}{ (c) Performance for IIT Delhi database } \\
Palm code [9] & 99.68 & 5.2108 & 1.800 \\
Ordinal code [21] & 100.00 & 1.1762 & 2.2174 \\
Proposed & $\mathbf{1 0 0 . 0 0}$ & $\mathbf{1 . 0 2 1 7}$ & $\mathbf{3 . 0 1 6 8}$ \\
\hline
\end{tabular}

TABLE 2: Computation time for key processes both local and global combination.

\begin{tabular}{lccc}
\hline & Extraction $(\mathrm{ms})$ & Matching $(\mathrm{ms})$ & Total $(\mathrm{ms})$ \\
\hline & (a) Systems speed for PolyU database & \\
Proposed & 54.56 & 1.5 & 56.06 \\
\hline \multirow{4}{*}{ (b) Systems speed for COEP database } \\
Proposed & 85.41 & 3.07 & 88.48 \\
\hline \multirow{4}{*}{ Proposed } & (c) Systems speed for IIT Delhi database \\
\hline
\end{tabular}

number is assigned to every user for the acquired images. The resolution of these images is $800 \times 600$ pixels. After preprocessing, original images, $150 \times 150$ pixels, are automatically cropped and considered as ROI.

Verification means by evaluating one or more individual genetic traits of a person can be uniquely identified. In this paper, all the classes of palmprint images were involved. The metrics such as EER, correct recognition rate (CRR), and receiver operating characteristics (ROC) are used to measure the recognition accuracy. The CRR of a system can be defined by

$$
\mathrm{CRR}=\frac{R 1}{R 2} \times 100,
$$

where $R 1$ denotes the number of correct recognition of palmprint images and $R 2$ is the total number of palmprint images. Performance comparison of different palmprint verification schemes is shown in Table 1 . Table 2 shows the computation time for key processes.

ROC curves are used to analyse the performance of the palmprint biometric system. ROC is a plot of genuine acceptance rate (GAR) against false acceptance rate (FAR) and shown in Figures 11, 12, 13, 14, 15, 16, 17, 18, and 19 for PolyU palmprint database, COEP palmprint database, and IIT Delhi palmprint database. The FAR and FRR of a palmprint biometric system can be obtained by adjusting the various thresholds. It is known that both FAR and FRR are 


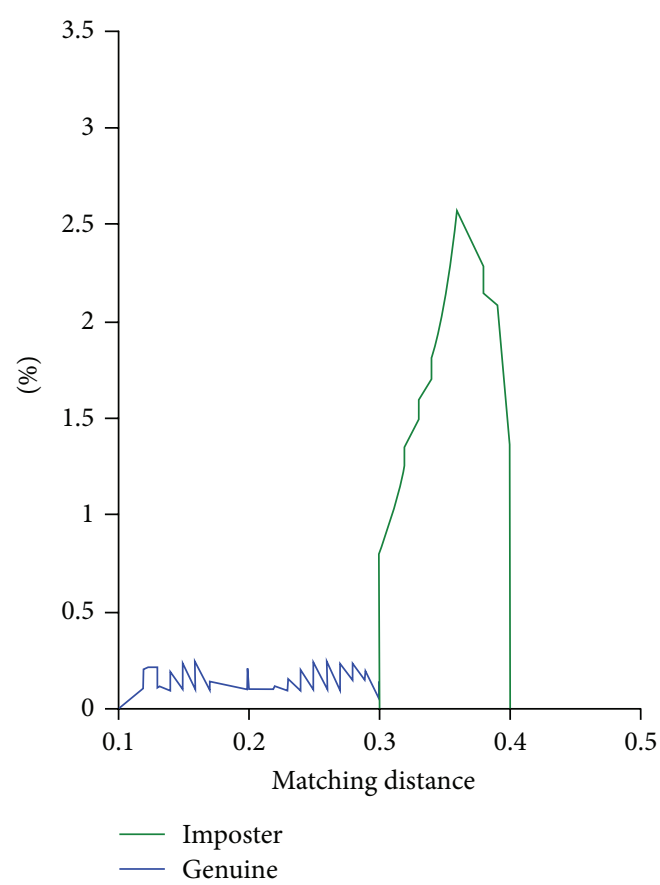

FIGURE 21: Distance distributions of genuine matching and imposter matching of proposed DOST for COEP palmprint database.

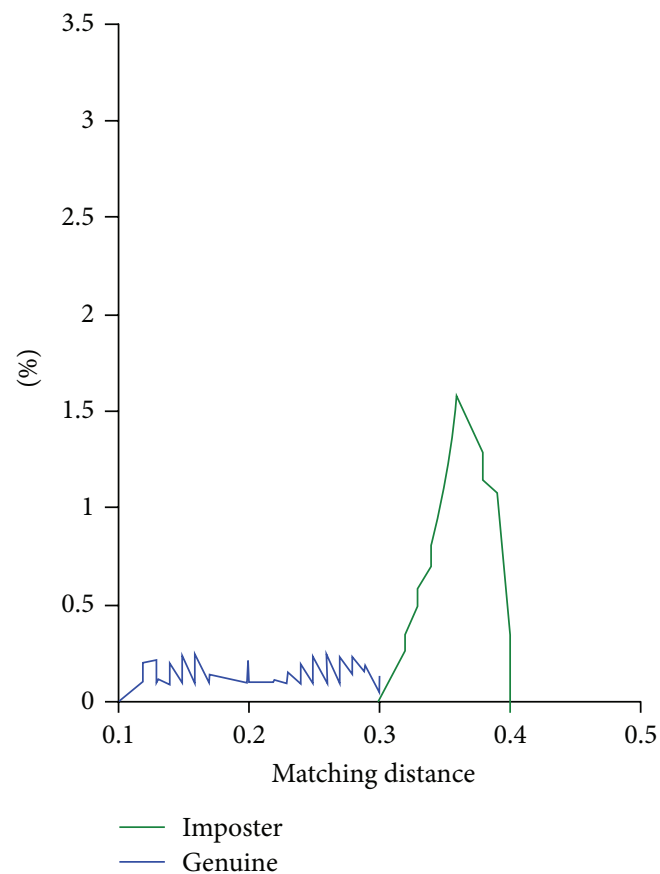

FIGURE 22: Distance distributions of genuine matching and imposter matching of proposed DOST for IIT Delhi palmprint database.

inversely related. Thus, at a particular threshold, these two curves intersect. This intersect point is termed as an EER. The EER is defined at the FAR which is equal to the FRR. The distance distributions of genuine matching and imposter matching acquired by proposed scheme are plotted in Figures 20,21 , and 22 . The decidability index (DI) is defined as how well the genuine and the imposter distributions are separated which is defined as

$$
\mathrm{DI}=\frac{\left|\mu_{1}-\mu_{2}\right|}{\sqrt{\left(\sigma_{1}^{2}+\sigma_{2}^{2}\right) / 2}},
$$

where $\mu_{1\left(\mu_{2}\right)}$ is the mean of the genuine (imposter) matching distances and $\sigma_{1}\left(\sigma_{2}\right)$ is the standard deviation of the genuine (imposter) matching distances. Then performance of any biometric system can be measured by its $d^{\prime}$ score among other metrics.

\section{Conclusion}

In this work, palmprint images are used for recognition. LGIF based palmprint features are critical for the image recognition and observation. They play dissimilar and corresponding roles in a system. In LGIF, the local instantaneous phase information obtained by the DOST technique is measured as the local feature. From the perception of time-frequency analysis, if the scale of the DOST goes to infinity, it converts to the Fourier transform. The global information of palmprint recognition system refines the alignment of palmprint image in pattern matching. Extensive investigational outcomes shown on the palmprint database indicate that the proposed system could achieve much better performance in terms of EER.

\section{Conflict of Interests}

The authors declare that there is no conflict of interests regarding the publication of this paper.

\section{Acknowledgments}

The authors would like to thank Hong Kong Polytechnic University, IIT-Delhi, and College of Engineering-Pune for providing the palmprint databases.

\section{References}

[1] A. K. Jain, P. J. Flynn, and A. Ross, Handbook of Biometrics, Springer, Berlin, Germany, 2010.

[2] D. Maltoni, D. Maio, A. K. Jain, and S. Prabhakar, Handbook of Fingerprint Recognition, Springer, London, UK, 2009.

[3] N. Ratha and R. Bolle, Automatic Fingerprint Recognition Systems, Springer, New York, NY, USA, 2011.

[4] R. Belguechi, E. Cherrier, C. Rosenberger, and S. Ait-Aoudia, "Operational bio-hash to preserve privacy of fingerprint minutiae templates," IET Biometrics, vol. 2, no. 2, pp. 76-84, 2013.

[5] J. Gu, J. Zhou, and C. Yang, "Fingerprint recognition by combining global structure and local cues," IEEE Transactions on Image Processing, vol. 15, no. 7, pp. 1952-1964, 2006.

[6] F. Yuchun, T. Tieniu, and W. Yunhong, "Fusion of global and local features for face verification," in Proceedings of the International Conference on Pattern Recognition, pp. 382-385, Quebec, Canada, August 2002.

[7] K. Miyazawa, K. Ito, T. Aoki, K. Kobayashi, and H. Nakajima, "An effective approach for Iris recognition using phase-based 
image matching," IEEE Transactions on Pattern Analysis and Machine Intelligence, vol. 30, no. 10, pp. 1741-1756, 2008.

[8] Z. Sun, Y. Wang, T. Tan, and J. Cui, "Improving iris recognition accuracy via cascaded classifiers," IEEE Transactions on Systems, Man and Cybernetics C: Applications and Reviews, vol. 35, no. 3, pp. 435-441, 2005.

[9] D. Zhang, W.-K. Kong, J. You, and M. Wong, "Online palmprint identification," IEEE Transactions on Pattern Analysis and Machine Intelligence, vol. 25, no. 9, pp. 1041-1050, 2003.

[10] C. C. Han, H. L. Cheng, C. L. Lin, and K. C. Fan, "Personal authentication using palm-print features," Pattern Recognition, vol. 36, no. 2, pp. 371-381, 2003.

[11] H. Matos, P. Oliveira, and F. Magalhaes, "Hand-geometry based recognition system," in Image Analysis and Recognition, vol. 7325 of Lecture Notes in Computer Science, pp. 38-45, Springer, 2012.

[12] D. Huang, Y. Tang, Y. Wang, L. Chen, and Y. Wang, "Hand vein recognition based on oriented gradient maps and local feature matching," in Computer Vision, vol. 7727 of Lecture Notes in Computer Science, pp. 430-444, Springer, New York, NY, USA, 2013.

[13] L. Nanni and A. Lumini, "A multi-matcher system based on knuckle-based features," Neural Computing and Applications, vol. 18, no. 1, pp. 87-91, 2009.

[14] A. Kumar and Y. Zhou, "Personal identification using finger knuckle orientation features," Electronics Letters, vol. 45, no. 20, pp. 1023-1025, 2009.

[15] H. Hollein, Forensic Voice Identification, Academic Press, 2002.

[16] C. Middendorff, Multi-Biometric Approaches to Ear Biometrics and Soft Biometrics, BiblioBazaar, 2011.

[17] C. McCool, R. Wallace, M. McLaren, L. El Shafey, and S. Marcel, "Session variability modelling for face authentication," IET Biometrics, vol. 2, no. 3, pp. 117-129, 2013.

[18] T. H. Nguyen, Y. Wang, Y. Ha, and R. Li, "Improved chaff point generation for vault scheme in bio-cryptosystems," IET Biometrics, vol. 2, no. 2, pp. 48-55, 2013.

[19] N. Duta, A. K. Jain, and K. V. Mardia, "Matching of palmprints," Pattern Recognition Letters, vol. 23, no. 4, pp. 477-485, 2002.

[20] J. Funada, N. Ohta, M. Mizoguchi et al., "Feature extraction method for palmprint considering elimination of creases," in Proceedings of the International Conference on Pattern Recognition, pp. 1849-1854, Brisbane, Australia, August 1998.

[21] Z. N. Sun, T. Tan, Y. Wang, and S. Z. Li, "Ordinal palmprint represention for personal identification [represention read representation]," in Proceedings of the IEEE Computer Society Conference on Computer Vision and Pattern Recognition (CVPR '05), vol. 1, pp. 279-284, June 2005.

[22] Y. Su, S. Shan, X. Chen, and W. Gao, "Hierarchical ensemble of global and local classifiers for face recognition," IEEE Transactions on Image Processing, vol. 18, no. 8, pp. 1885-1896, 2009.

[23] F. Li, K. H. Leung, and X. Yu, "A two-level matching scheme for speedy and accurate palmprint identification," in Advances in Multimedia Modeling, vol. 4352 of Lecture Notes in Computer Science, pp. 323-332, Springer, Berlin, Germany, 2006.

[24] X. Pan, Q. Ruan, and Y. Wang, "Palmprint recognition using fusion of local and global features," in Proceedings of the International Conference on Intelligence Signal Processing and Communication System, pp. 642-645, Xiamen, China, November 2007.
[25] S. B. Nikam, P. Goel, R. Tapadar, and S. Agarwal, "Combining gabor local texture pattern and wavelet global features for fingerprint matching," in Proceedings of the International Conference on Computational Intelligence and Multimedia Applications (ICCIMA '07), pp. 409-416, Washington, DC, USA, 2007.

[26] G. S. Badrinath and P. Gupta, "Stockwell transform based palmprint recognition," Applied Soft Computing, vol. 11, no. 7, pp. 4267-4281, 2011.

[27] "PolyU Palmprint Database," The Hong Kong Polytechnic University, July 2010, http://www4.comp.polyu.edu.hk/ biometrics.

[28] COEP Palm Print Database (College of Engineering Pune), http://www.coep.org.in/.

[29] “Palmprint Image Database version 1," IIT, Delhi, India, 2007, http://www4.comp.polyu.edu.hk/ csajaykr/IITD/Database Palm.htm. 


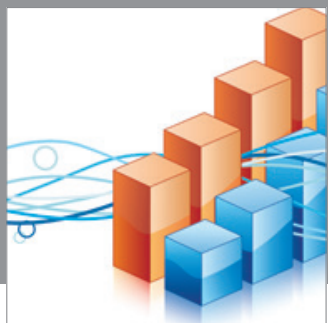

Advances in

Operations Research

mansans

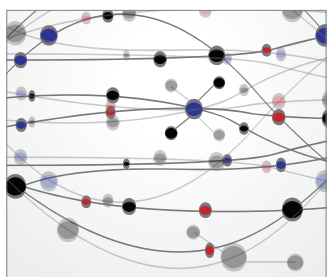

The Scientific World Journal
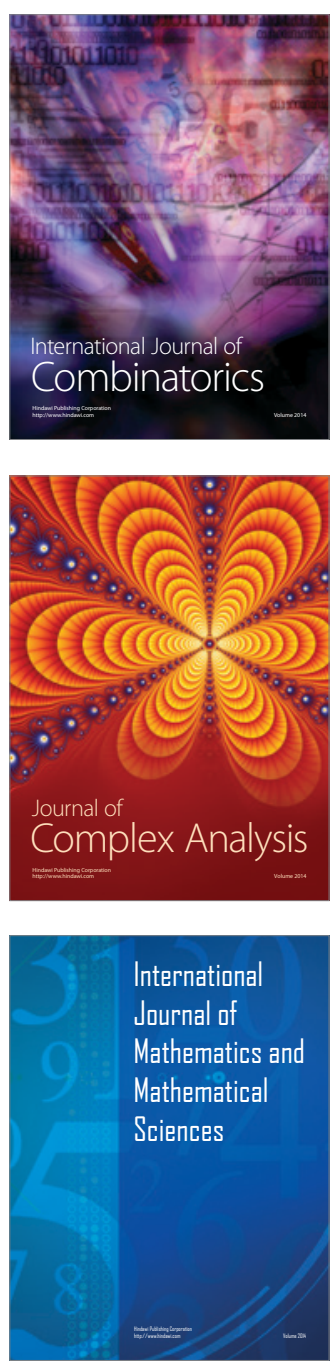
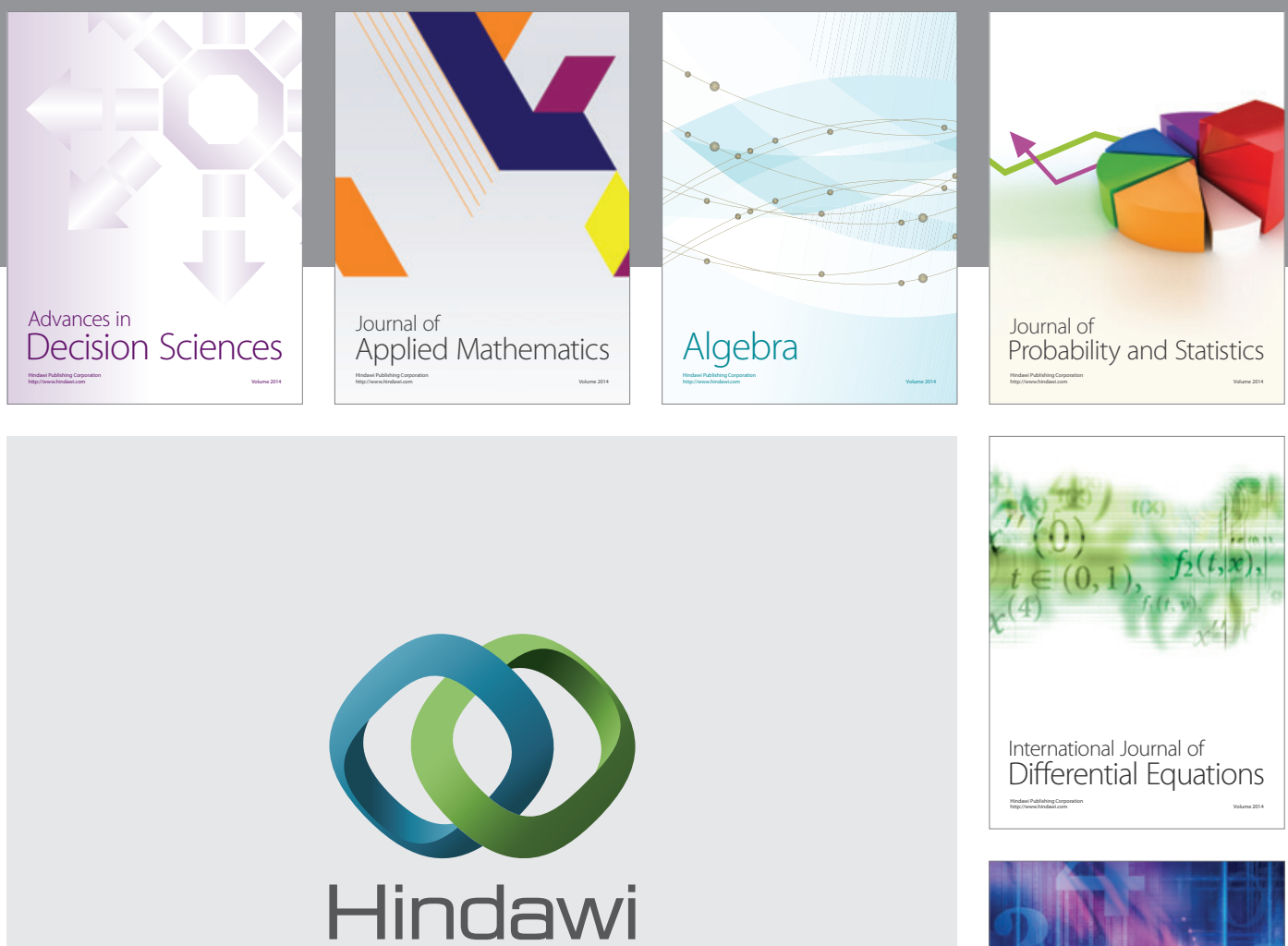

Submit your manuscripts at http://www.hindawi.com
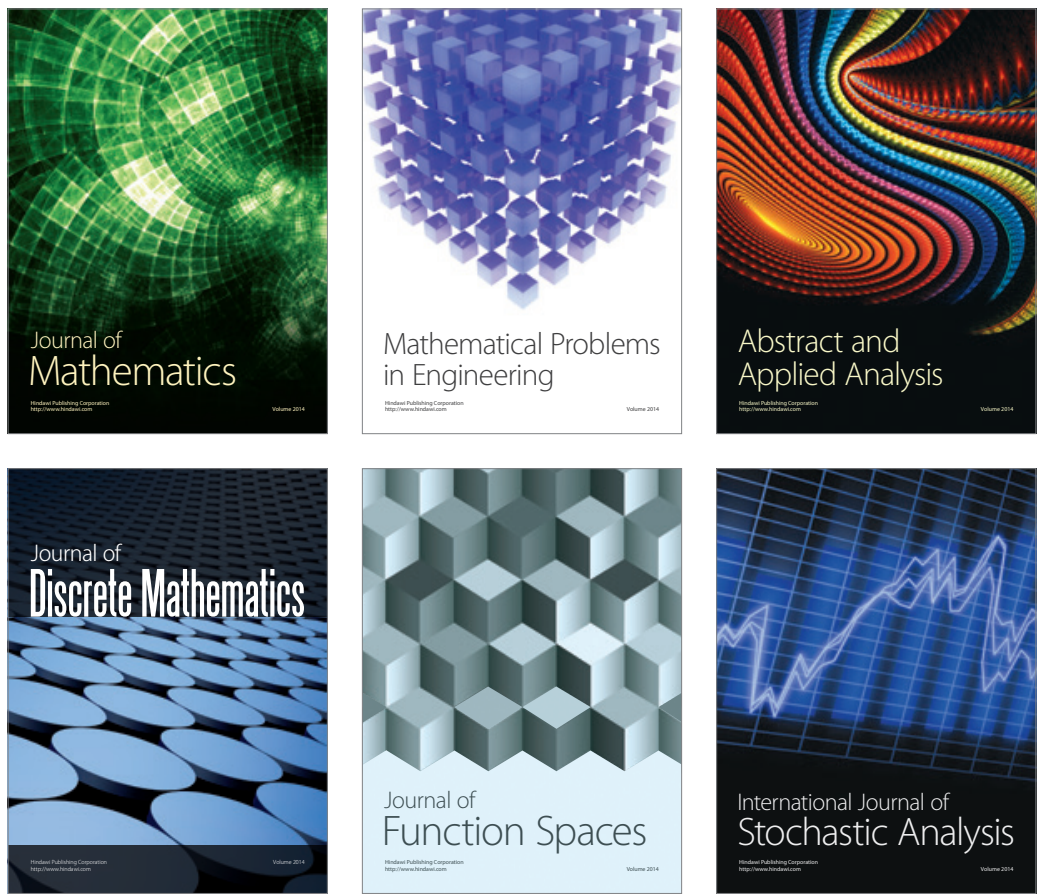

Journal of

Function Spaces

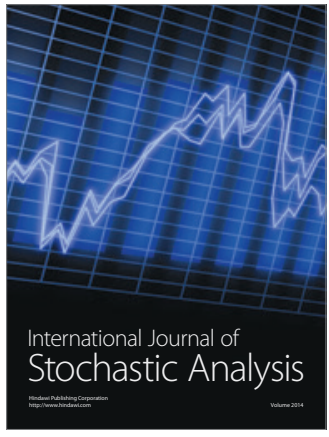

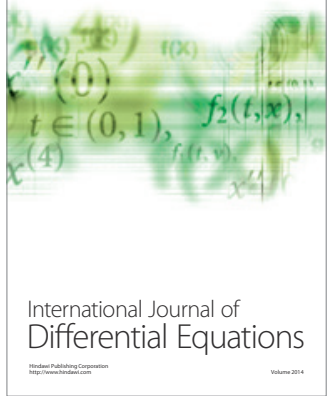
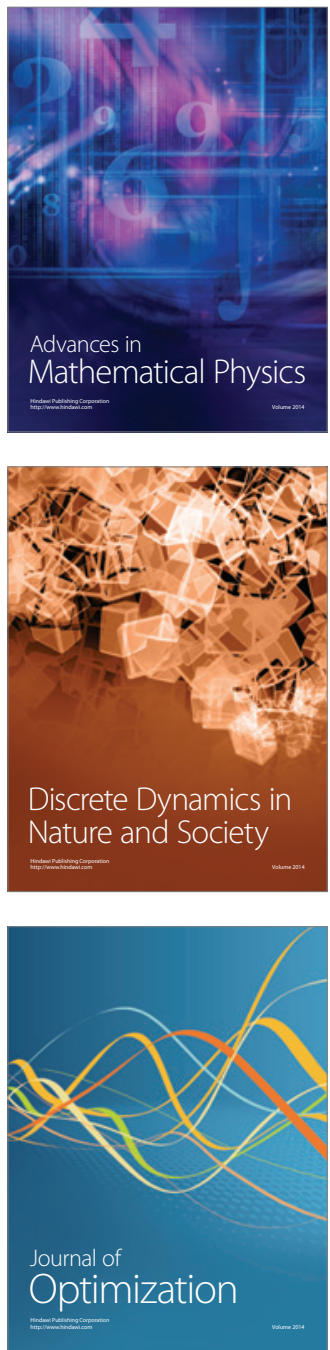